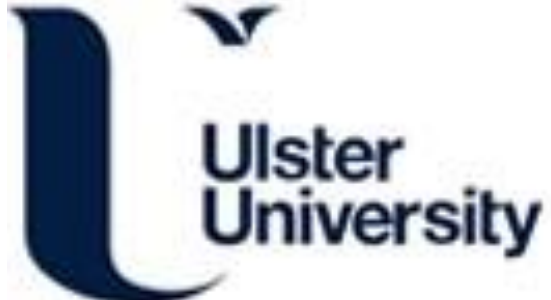

\section{Solution Self-Assembly of the Sophorolipid Biosurfactant and Its Mixture with Anionic Surfactant Sodium Dodecyl Benzene Sulfonate}

Penfold, J., Chen, ML., Thomas, RK., Dong, CC., Smyth, TJP., Perfumo, A., Marchant, R., Banat, IM., Stevenson, P., Parry, A., Tucker, I., \& Grillo, I. (2011). Solution Self-Assembly of the Sophorolipid Biosurfactant and Its Mixture with Anionic Surfactant Sodium Dodecyl Benzene Sulfonate. Langmuir, 27, 8867-8877.

Link to publication record in Ulster University Research Portal

Published in:

Langmuir

Publication Status:

Published (in print/issue): 01/06/2011

\section{Document Version}

Publisher's PDF, also known as Version of record

\section{General rights}

Copyright for the publications made accessible via Ulster University's Research Portal is retained by the author(s) and / or other copyright owners and it is a condition of accessing these publications that users recognise and abide by the legal requirements associated with these rights.

\section{Take down policy}

The Research Portal is Ulster University's institutional repository that provides access to Ulster's research outputs. Every effort has been made to ensure that content in the Research Portal does not infringe any person's rights, or applicable UK laws. If you discover content in the Research Portal that you believe breaches copyright or violates any law, please contact pure-support@ulster.ac.uk. 


\title{
Solution Self-Assembly of the Sophorolipid Biosurfactant and Its Mixture with Anionic Surfactant Sodium Dodecyl Benzene Sulfonate
}

\author{
Jeff Penfold, ${ }^{*,+, \neq}$ Minglei Chen, ${ }^{\dagger}$ Robert K. Thomas, ${ }^{\dagger}$ Chuchuan Dong, ${ }^{\dagger}$ Thomas J. P. Smyth, $^{\S}$ \\ Amedea Perfumo, ${ }^{\S}$ Roger Marchant, ${ }^{\S}$ Ibrahim M. Banat, ${ }^{\S}$ Paul Stevenson, " Alyn Parry," \\ Ian Tucker," and I. Grillo ${ }^{\perp}$ \\ ${ }^{\dagger}$ Physical and Theoretical Chemistry Department, University of Oxford, South Parks Road, Oxford, U.K. \\ ${ }^{\ddagger}$ ISIS, STFC, Rutherford Appleton Laboratory, Chilton, Didcot, Oxon, U.K. \\ ${ }^{\S}$ School of Biomedical Sciences, University of Ulster, Coleraine, Northern Ireland \\ "Unilever Research and Development Laboratory, Port Sunlight, Wirral, U.K. \\ ${ }^{\perp}$ Institut Laue Langevin, 6 rue Jules Horowitz, F-38042, Grenoble, Cedex 09, France
}

Supporting Information

ABSTRACT: The self-assembly in aqueous solution of the acidic (AS) and lactonic (LS) forms of the sophorolipid biosurfactant, their mixtures, and their mixtures with anionic surfactant sodium dodecyl benzene sulfonate, LAS, has been studied using predominantly small-angle neutron scattering, SANS, at relatively low surfactant concentrations of $<30 \mathrm{mM}$. The more hydrophobic lactonic sophorolipid forms small unilamellar vesicles at low surfactant concentrations, in the concentration range of 0.2 to $3 \mathrm{mM}$, and transforms via a larger unilamellar vesicle structure at $7 \mathrm{mM}$ to a disordered dilute phase of tubules at higher concentrations, 10 to $30 \mathrm{mM}$. In marked contrast, the acidic sophorolipid is predominantly in the form of small globular micelles in the concentration range of 0.5 to $30 \mathrm{mM}$, with a lower concentration of larger, more planar aggregates (lamellar or vesicular) in coexistence. In mixtures of AS and LS, over the same concentration range, the micellar structure associated with the AS sophorolipid dominates the mixed-phase behavior. In mixtures of anionic surfactant LAS with the AS sophorolipid, the globular micellar structure dominates over the entire

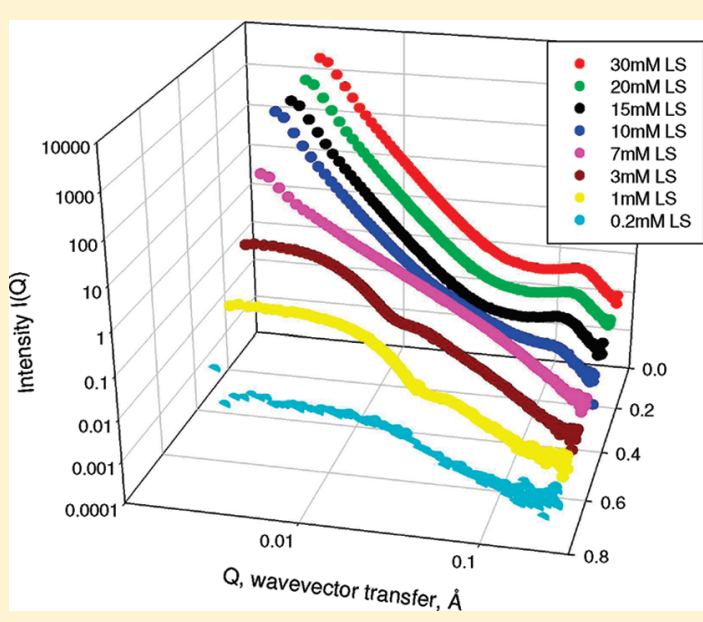
composition and concentration range studied. In contrast, mixtures of LAS with the LS sophorolipid exhibit a rich evolution in phase behavior with solution composition and concentration. At low surfactant concentrations, the small unilamellar vesicle structure present for LS-rich solution compositions evolves into a globular micelle structure as the solution becomes richer in LAS. At higher surfactant concentrations, the disordered lamellar structure present for LS-rich compositions transforms to small vesicle/lamellar coexistence, to lamellar/micellar coexistence, to micellar/lamellar coexistence, and ultimately to a pure micellar phase as the solution becomes richer in LAS. The AS sophorolipid surfactant exhibits self-assembly properties similar to those of most other weakly ionic or nonionic surfactants that have relatively large headgroups. However, the more hydrophobic nature of the lactonic sophorolipid results in a more complex and unusual evolution in phase behavior with concentration and with concentration and composition when mixed with anionic surfactant LAS.

\section{INTRODUCTION}

Surfactants are used in a wide range of different applications, ${ }^{1}$ and currently the large majority of surfactants used are petroleum-based or are from other nonsustainable sources. The use of synthetic surfactants is under increasing pressure because of their environmental impact, and there is increasing interest in the use of biosurfactants because of their biosustainable and biodegradable potential. Biosurfactants are produced by microorganisms, and their surface activity properties are broadly similar to conventional surfactants, resulting in reduced surface/interfacial tension, wetting, and emulsification. ${ }^{2}$ Biosurfactants do, however, have a number of key advantages over their synthetic counterparts, which include lower toxicity, antimicrobial properties, greater biodegradability, ${ }^{3}$ better environmental compatibility, ${ }^{4}$ high selectivity, specific activity at extreme temperatures, $\mathrm{pH}$, and salinity, ${ }^{5,6}$ and the ability to be produced from renewable feedstocks. ${ }^{7}$ Biosurfactants, like their conventional counterparts, are also amphiphilic in nature; the hydrophobic building blocks

\section{Received: May 5, 2011}

Revised: June 3, 2011

Published: June 06, 2011 

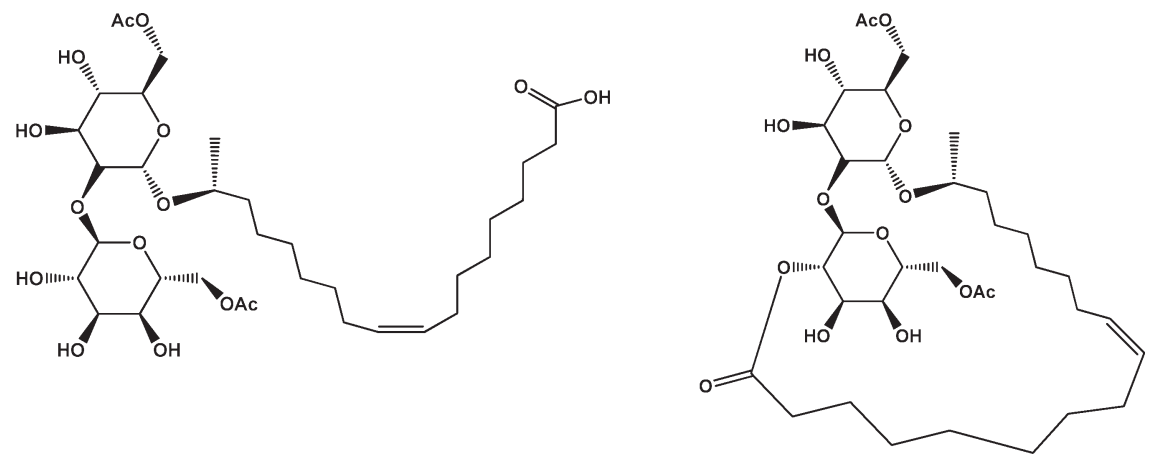

Figure 1. Chemical structure of the acidic sophorolipid (AS) and the lactonic sophorolipid (LS).

are fatty acids and fatty alcohols, and the hydrophilic parts are mainly carbohydrates and amino acids/peptides. Biosurfactants can be classified into high-molecular-weight and low-molecularweight molecules, ${ }^{8}$ and typical examples of biosurfactants of low molecular weight are the glycolipids, such as rhamnolipids, sophorolipids, trehalolipids, and the lipopeptides, such as surfactin. The glycolipids are the best known and most commonly studied of the lower-molecular-weight biosurfactants. In broad terms, they are formed from carbohydrates, in combination with long-chain aliphatic acids or hydroxyaliphatic acids. Despite considerable progress made in studying the production of biosurfactants, there is a lack of understanding of many of their fundamental physicochemical properties. Very few papers have reported the surface and solution behavior of biosurfactant and biosurfactant/surfactant mixtures in any detail.

We have used neutron reflectivity, SANS, and surface tension to investigate in detail the surface adsorption and solution selfassembly of another type of biosurfactant, rhamnolipids R1 and $\mathrm{R} 2{ }^{9}$ and their mixtures with an anionic surfactant, sodium 6-dodecyl benzene sulfonate, LAS. ${ }^{10}$ More recently, we have investigated the surface adsorption behavior of another glycolipid, sophorolipids LS and AS, and their mixtures with anionic surfactant LAS, determined predominantly by NR and ST. ${ }^{11}$ In this article, the solution self-assembly of sophorolipids, LS and AS, and their mixtures with LAS will be presented and discussed.

There are two main structural variations of the sophorolipids, ${ }^{12}$ determined by the nature of the hydrophobic moiety (lactone or free acid form) and the extent of esterification, and the two dominant sophorolipid structures are shown in Figure 1.

The hydrophobic moiety is predominantly hydroxy-oleic acid, and the headgroup consists of a sophorose unit.

Using light microscopy and small- and wide-angle X-ray scattering techniques, Zhou et al. ${ }^{13}$ reported the formation of giant helical ribbon structures by nonacetylated acidic sophorolipids under acidic conditions at $\mathrm{pH}$ values from 2.0 to 4.1. They attributed the formation of such ribbons to the unique sophorolipid molecular structure arising from the highly asymmetrical polar heads, the kinked hydrophobic chains, and the nonamide polar-nonpolar linkages. At neutral $\mathrm{pH}$, they observed the formation of a clear AS solution, which was assumed to be in the form of micelles. Zhang et al. ${ }^{14}$ investigated the effect of alkyl ester chain length on the surfactant $\mathrm{cmc}$. They found that the $\mathrm{cmc}$ for sophorolipid esters decreases by $50 \%$ for each additional $\mathrm{CH}_{2}$ group attached to the carboxyl end of the alkyl ester. The extent of esterification, by either acetylation or lactonization, also determines the solubility of the final product. Otto et al. ${ }^{15}$ reported that crude sophorolipid mixtures had a water solubility of 2 to $3 \mathrm{~g} \mathrm{~L}^{-1}$.
Table 1. Sample Composition of Four Different AS Samples Determined by HPLC-MS

\begin{tabular}{lcccl} 
& $\begin{array}{c}\text { diacetylated } \\
\text { AS (\%) }\end{array}$ & $\begin{array}{c}\text { monoacetylated } \\
\text { AS (\%) }\end{array}$ & $\begin{array}{c}\text { nonacetylated } \\
\text { AS (\%) }\end{array}$ & $\begin{array}{l}\text { LS } \\
(\%)\end{array}$ \\
\hline AS(S1) & 89.4 & 2.2 & 0 & 8.4 \\
AS(S2) & 13.6 & 66.5 & 7.9 & 12.0 \\
AS(S3) & 0 & 0 & 93.2 & 6.8 \\
AS(S4) & 8.5 & 39.0 & 52.5 & 0 \\
\hline
\end{tabular}

In contrast, purified diacetylated C18/1 LS is much less watersoluble (with a maximum solubility of $70 \mathrm{mg} \mathrm{L}^{-1}$ ). They also investigated the impact of the level of acetylation on the surface behavior of sophorolipids, and they found that monoacetylated $\mathrm{C} 18 / 1 \mathrm{LS}$ is less surface-active and has a higher surface tension of $40 \mathrm{mN} \mathrm{m}^{-1}$ at the $\mathrm{cmc}$ than does the diacetylated form.

\section{EXPERIMENTAL DETAILS}

Materials and Measurements Made. The sophorolipids, Sopholiance S, were purchased from Soliance Co. (France) and were separated into pure lactonic (LS) and acidic sophorolipid (AS) using MPLC, as described in detail elsewhere. ${ }^{9,10,16}$ It was not possible to obtain pure AS (with two acetyl groups) without the presence of a trace amount of LS (with no acetyl groups). There are four different types of AS samples, and for clarity, the detailed compositions of those different AS samples are listed in Table 1.

Compared with the rhamnolipids, ${ }^{9}$ the HPLC-MS analysis revealed a much more complicated mixture for each of the sophorolipids. The different structural variations include the length of the fatty acid chain $\left(\mathrm{C}_{16}-\mathrm{C}_{22}\right)$, the number of double bonds on the chain $(0-2)$, and the number of acetyl groups attached to the sophorose sugar headgroup $(0-2)$. For the separated h-LS, 13 different homologues were identified, and among these, $87 \%$ (mole ratio) were LS with two acetyl groups and $13 \%$ were LS with one acetyl group. The most abundant structure is $\mathrm{C}_{18: 1}$ (one double bond) with two acetyl groups, and this accounts for $54 \%$ of all of the LS mixtures.

Sodium 6-dodecyl benzene-4 sulfate, LAS, (6-phenyl isomer) was prepared, purified, and characterized as described in detail elsewhere. ${ }^{17}$ The SANS measurements were made at relatively low surfactant concentration, $<30 \mathrm{mM}$ (because of the relatively low solubility of the sophorolipids), and in $\mathrm{D}_{2} \mathrm{O}$ at $30^{\circ} \mathrm{C}$ and using hydrogenous sophorolipids.

For binary surfactant mixtures, measurements were made for four different LS/AS mixtures consisting of LS (diacetylated) mixed in turn with four different types of AS (AS(S1), diacetylated-AS; AS(S2), monoacetylated-AS; AS(S3), nonacetylated-AS; and AS(S4), nonacetylated-AS). The AS(S1) to AS(S3) samples all contain trace amounts of LS, whereas the AS(S4) sample is pure AS. Taking into account the finite 
LS component present restricted the composition range over which AS/LS measurements could be made. The same LS (diacetylated) sample was used in the LS/LAS mixture measurement. For AS/LAS mixtures, only AS(S4) (nonacetylated AS) was used. For LS/LAS mixtures, the SANS measurements were made over a composition range from 90:10 to 10:90 at solution concentrations from 3 to $30 \mathrm{mM}$. For LS/AS(S1) and AS(S4)/LAS mixtures, SANS measurements were made over a composition range from 80:20 to 10:90 and for solution concentrations from 5 to $30 \mathrm{mM}$.

SANS measurements were also made to determine the phase behavior of ternary surfactant mixtures of LS (diacetylated), AS(S1) (diacetylated), and LAS. A range of solution compositions of sophorolipid/LAS from 80:20 to 20:80 were measured at solution concentrations from 5 to $30 \mathrm{mM}$ for three different LS/AS compositions (2:1, 1:1, and $1: 2$ ).

Small-Angle Neutron Scattering, SANS. The solution selfassembly and phase behavior of the sophorolipids have been determined predominantly from the SANS measurements. Dynamic light scattering and optical birefringence measurements using crossed polarizers were also used to assist in the characterization of the phase behavior but are not described in any detail here.

The SANS measurements were made on the D22 and D11 diffractometers at the ILL, France, ${ }^{18,19}$ and on the LOQ and SANS2D ${ }^{20,21}$ diffractometers at ISIS, UK. On D22, the measurements were made at a neutron wavelength, $\lambda$, of $8 \AA$, at $\Delta \lambda / \lambda$ of $10 \%$, and at two sample-todetector distances, 3.5 and $16.5 \mathrm{~m}$, to cover a scattering vector, $Q$ range of 0.002 to $0.2 \AA^{-1}$ (where $Q=4 \pi / \lambda \sin \left({ }^{\theta} / 2\right)$ and $\theta$ is the scattering angle). The D11 measurements were made at a neutron wavelength, $\lambda$, of $6 \AA$, a $\Delta \lambda / \lambda$ of $10 \%$, and three sample-to-detector distances, 1.1, 5.0, and $16.5 \mathrm{~m}$, to cover a scattering vector, $Q$ range of 0.003 to $0.25 \AA^{-1}$. On LOQ, the measurements were made using the white beam time-offlight method using neutron wavelengths in the range 2 to $10 \AA$ and a sample-to-detector distance of $4 \mathrm{~m}$ to cover a $Q$ range of 0.008 to 0.25 $\AA^{-1}$. All of the LOQ measurements were made with 8-mm-diameter beam and on D11 and D22 using a beam of $7 \times 10 \mathrm{~mm}^{2}$. The measurements on SANS2D were made using the white beam time-offlight method with neutron wavelengths of $\sim 2-12 \AA$ and a $6 \mathrm{~m}$ sampleto-detector distance to cover a $Q$ range of $0.01-0.35 \AA$. The data were corrected for background scattering, detector response, and the spectral distribution of the incident beam (for LOQ and SANS2D) and converted to an absolute scattering cross section, $(\mathrm{d} \sigma) /(\mathrm{d} \Omega)$ (in $\left.\mathrm{cm}^{-1}\right)$ using standard procedures. ${ }^{22,23}$

The form of the SANS scattering patterns ( $Q$ dependence) was used qualitatively to identify the lamellar (vesicle), micellar, and mixed-phase regions of the overall phase behavior. In the purely micellar regions, a detailed quantitative analysis was also made using standard modeling procedures for mixed surfactant micelles ${ }^{24}$ adapted for the sophorolipids. In the small-vesicle region observed for LS and LS/LAS mixtures, the same basic core + shell model was used but with different molecular constraints. The larger lamellar/vesicle structures were not analyzed quantitatively.

The scattering from globular surfactant micelles in aqueous solution is described by the decoupling approximation, derived by Hayter and Penfold, ${ }^{24}$ such that

$$
\frac{\mathrm{d} \sigma}{\mathrm{d} \Omega}=n\left[S(Q)\left|\langle F(Q)\rangle_{Q}\right|^{2}+\left\langle|F(Q)|^{2}\right\rangle_{Q}-\left|\langle F(Q)\rangle_{Q}\right|^{2}\right]
$$

where the averages denoted by $\langle Q\rangle$ are averages over particle size and orientation, $n$ is the micelle number density, $S(Q)$ is the intermicellar structure factor, and $F(Q)$ is the micelle form factor. The micelle structure (form factor, $F(Q)$ ) is modeled using a standard core-andshell model, ${ }^{24}$ where the form factor for globular micelles is

$$
F(Q)=V_{1}\left(\rho_{1}-\rho_{2}\right) F_{0}\left(Q r_{1}\right)+V_{2}\left(\rho_{2}-\rho_{\mathrm{s}}\right) F_{0}\left(Q r_{2}\right)
$$

and $r_{1}$ and $r_{2}$ are the core and shell radii, $V_{i}=4 \pi r_{i}^{3} / 3$,

$$
F_{0}\left(Q R_{i}\right)=\frac{3 j_{1}\left(Q r_{i}\right)}{Q r}=\frac{3[\sin (Q r)-Q r \cos (Q r)]}{(Q r)^{3}}
$$

$\rho_{1}, \rho_{2}$, and $\rho_{\mathrm{s}}$ are the scattering-length densities of the micelle core and shell and of the solvent, and $j_{1}\left(Q r_{i}\right)$ is a first-order spherical Bessel function. The decoupling approximation assumes that for interacting (finite $S(Q)$ ) globular micelles there is no correlation among position, size, and orientation. The structure factor, $S(Q)$, which quantifies the intermicellar interactions/correlations, is included using the rescaled mean spherical approximation, RMSA, calculation ${ }^{25}$ for a repulsive screened Coulombic intermicellar interaction potential characterized by the surface charge of the micelle, $z$, the Debye-Huckel inverse screening length, $\kappa_{\mathrm{dh}}$ (defined in the usual way), and the micelle number density, $n$.

A standard core + shell micelle form factor, in which molecular constraints are incorporated, is used to describe the micelle geometry ${ }^{24}$ and has been extensively and successfully used for a wide range of micellar systems, including the rhamnolipid biosurfactants. ${ }^{9,10}$ The form factor can be spherical or elliptical depending upon the geometrical constraints. For spheres, the inner core radius $R 1$ containing the alkyl chains is constrained to have a maximum dimension of the alkyl chain, $l_{c}$ (taken as $11.5 \AA$ for the sophorolipid and 14.0 $\AA$ for LAS). This is modified by an additional factor ext (such that $R 1=l_{c}$ ext) to allow for some variation in the packing constraints. In practice, here ext is in the range of 0.95 to 1.05 and so is not documented. The outer shell of radius $R 2$ contains the headgropups and associated hydration and is determined simply by space filling. For aggregation numbers, $v$, greater than that accommodated in a spherical volume defined by $R 1=l_{c}$, growth is accommodated by allowing the micelle shape to become elliptical. The ellipticity is defined by an ellipticity parameter, ee, such that the overall dimension are $R 2, R 2$, eeR2. From known molecular volumes and neutron scattering lengths, ${ }^{24}$ all of the parameters for eqs $1-3$ can be calculated, and the key refinable parametes are $v$ and $z$. In the mixed systems, ideal mixing is assumed and average values are taken.

Other Measurements. The dynamic light scattering, DLS, measurements were made using a Malvern Zetasizer Nano ZS, and the data were analyzed using the CONTIN approach. ${ }^{26}$ Visual inspections of the optical properties of the solutions were supplemented by the determination of any birefringence using crossed polarizers.

\section{RESULTS AND DISCUSSION}

Sophorolipids. Lactonic Sophorolipids. SANS measurements for the lactonic sophorolipids (diacetylated) were made at concentrations in the range from $0.2 \mathrm{mM}$ to $30 \mathrm{mM}$. Figure 2 shows the scattering data for the lactonic sophorolipids across the concentration range and demonstrates a rich pattern of evolution in the phase behavior with increasing concentration.

At lower concentrations of $0.2,1$, and $3 \mathrm{mM}$, the scattering data show a shallow minimum at an intermediate $Q$ value, which is not accounted for by a simple core-shell model for small globular micelles. The data indicate somewhat larger spherically symmetrical assemblies, larger than allowed by the packing constraints associated with simple micellar structure. Quantitative analysis shows that the data are consistent with small unilamellar vesicles, $L_{\mathrm{sv}}$. A slightly modified core-shell model (eqs $1-3$ ) was used to analyze the data in which the inner core comprises solvent $\left(\mathrm{D}_{2} \mathrm{O}\right.$ with a scattering-length density of 6.35 $\times 10^{-6} \AA^{-2}$ ) and the shell is a surfactant bilayer. Hence the key model parameters are inner and outer radii $R_{1}$ and $R_{2}$, respectively, the polydispersity, $\sigma$ (described as a Schultz distribution ${ }^{21}$ ), the surface charge, $z$, the scattering length density of the shell, $\rho$, and 


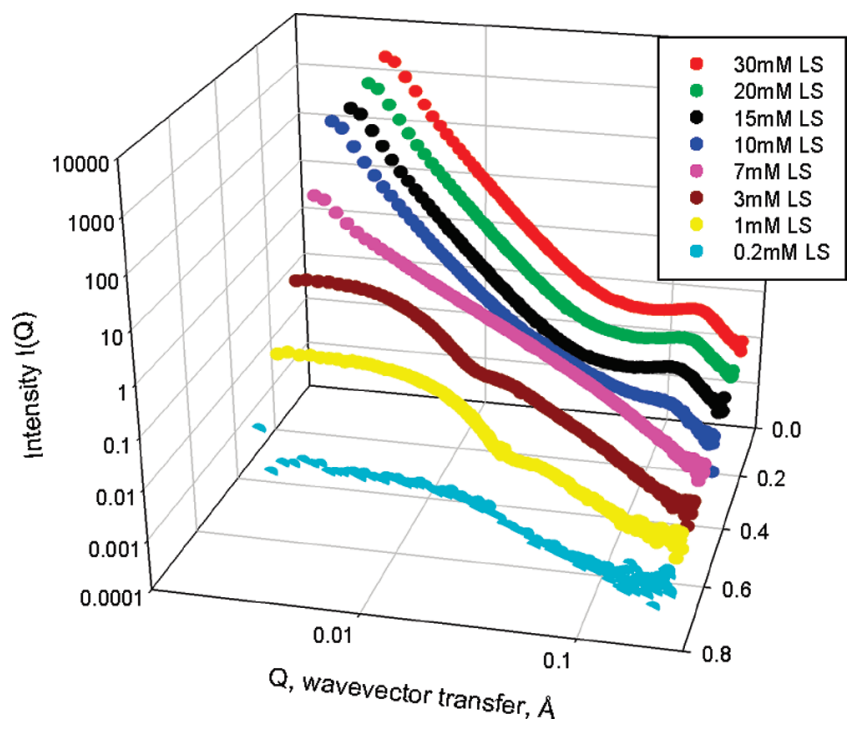

Figure 2. SANS data for the LS sophorolipid in $\mathrm{D}_{2} \mathrm{O}$ at different surfactant concentrations.

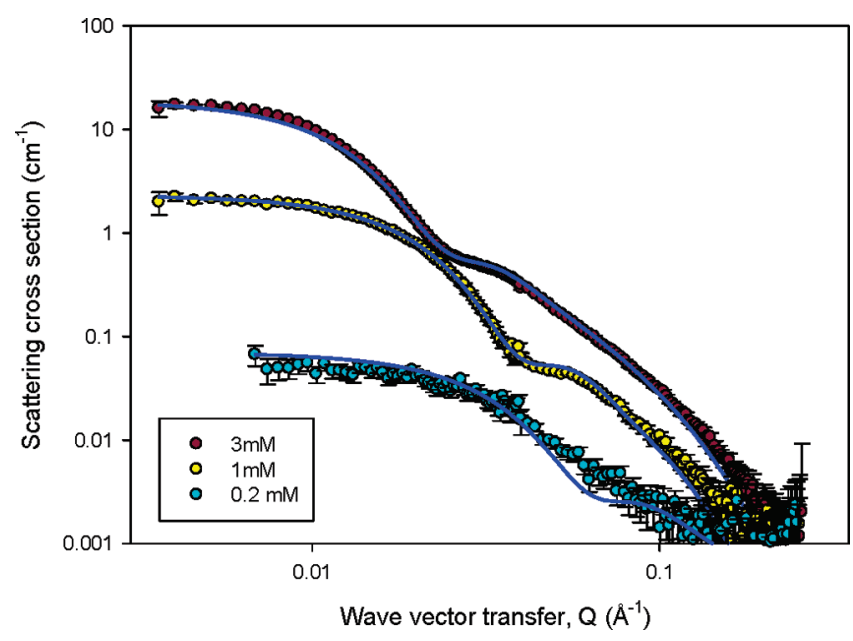

Figure 3. SANS data for the LS sophorolipid in $\mathrm{D}_{2} \mathrm{O}$ at surfactant concentrations of $0.2,1.0$, and $3.0 \mathrm{mM}$. The solid lines are core-shell model fits to the data for the small vesicle structure using the parameters summarized in Table 2.

the concentration of the vesicles. The concentration of vesicles and $\rho$ are constrained by the overall surfactant concentration and by assuming a space-filling bilayer. The refinable model parameters are then $R_{1}, R_{2}, \sigma$, and $z$. Figure 3 shows the model fits for the LS that form small vesicles at lower surfactant concentrations of $0.2,1$, and $3 \mathrm{mM}$, and the key model parameters are listed in Table 2.

The nanovesicle model provides a less-satisfactory description of the $0.2 \mathrm{mM}$ data, although it does encapsulate the main features of the scattering. A micelle model was not consistent with the data, and the coexistence of micelles and nanovesicles cannot be excluded.

The model parameters show that the vesicles grow with surfactant concentration, and the outer radius $R_{2}$ increases from $37 \AA$ at a concentration of $0.2 \mathrm{mM}$ to $104 \AA$ at $3 \mathrm{mM}$. The thickness of the surfactant bilayer is $15 \AA$ at concentration of $0.2 \mathrm{mM}$, and this increases to $24 \AA$ at the higher concentrations of
Table 2. Key Model Parameters for Core-Shell Model Analysis for Small Vesicle Structures for Diacetylated LS in $\mathrm{D}_{2} \mathrm{O}$

\begin{tabular}{crrcc} 
concentration $(\mathrm{mM})$ & $R_{1}(\AA)$ & $R_{2}(\AA)$ & polydispersity, $\sigma$ & charge, $z( \pm 1)$ \\
0.2 & $22 \pm 4$ & $37 \pm 8$ & $0.3 \pm 0.1$ & 1 \\
1 & $50 \pm 2$ & $74 \pm 4$ & $0.25 \pm 0.05$ & 1 \\
3 & $80 \pm 2$ & $104 \pm 4$ & $0.3 \pm 0.05$ & 1 \\
\hline
\end{tabular}

1 and $3 \mathrm{mM}$. Furthermore, these vesicles have a relatively small polydispersity $\sigma$ with a value of $0.25-0.30$. They are very weakly charged and consistent with the observation that LS is more nonionic than ionic in nature. ${ }^{11}$

Typical vesicle radii are generally much larger, $\gg 500 \AA^{24}$ Coldren et al. $^{26}$ reported vesicles formed in dodecyl trimethyl ammonium chloride (DTAC)/SDS mixtures with radii $>500 \AA$ with zero spontaneous curvature. Gonzales et al. ${ }^{27}$ studied the vesicle formation of LAS/imidazoline mixtures and observed unilamellar vesicles with radii of $\sim 150-360 \AA$ and a relatively large polydispersity of $0.35-0.60$. The formation of small, relatively monodisperse vesicles is less well known; however, a number of recent studies have reported small vesicle formation. ${ }^{26-30}$ Tucker et al. ${ }^{28}$ reported the formation of small nanovesicles with a mean diameter in the range of 70-140 $\AA$ and a low polydispersity of $\sim 0.2$ in dihexadecyl dimethyl ammonium bromide $\mathrm{DHDAB} / \mathrm{C}_{12} \mathrm{E}_{12}$, mixtures at intermediate surfactant composition with the addition of octanol or decanol. Grillo et al. ${ }^{29}$ have also reported similar relatively monodisperse small unilamellar vesicles in $\mathrm{DDAB} / \mathrm{C}_{12} \mathrm{E}_{4}$ mixtures, with an inner radius of $\sim 25-85 \AA$, a shell thickness of $23 \AA$, and a polydispersity of $<0.2$. Oberdisse et al. ${ }^{30}$ observed the formation of small vesicles in the system composed of Triton X100/octanol when a small amount of cetyl pyridinium chloride is added, with vesicle radii of $\sim 80-100 \AA$ and $\sigma<0.2$. The criteria for forming small vesicles with relatively narrow polydispersity have been discussed by Obserdisse et al. ${ }^{31,32}$ and Jung et al. ${ }^{33-35}$ and include a relatively high bending elasticity and a finite spontaneous curvature,

Surfactants with a packing parameter of between 0.5 and 1 are known to organize themselves into bilayers or vesicles according to the packing criteria of Israelachvili et al., ${ }^{36}$ where the packing parameter, pp, is defined as $\mathrm{pp}=V / \mathrm{Al}$, where $V$ is the alkyl chain volume, $l$ is the extended alkyl chain length, and $A$ is the area/ molecule. In the case of LS, the area per molecule from the neutron reflectivity data is $72 \AA^{2}$, hence the ratio of $V / l$ (volume of the chain/length of the chain) should be in the region between 36 and 72 to form vesicles. The volume and length of a straight $\mathrm{C}_{18}$ chain are $512 \AA^{3}$ and $24 \AA$, the ratio of which is 21 , which is not high enough for the formation of vesicles. Hence, to meet the criteria of Israelachivili et al. the chain cannot be fully extended. The hydrocarbon chain of LS is in the form of a highly bent lactone ring connected by both ends to the sophorolipid sugar headgroup, so using the fully extended chain length for a $C_{18}$ chain would not be appropriate. From the conformation illustrated in Figure 1, it is assumed that $l$ is $\sim 11 \AA$ (effectively a di$\mathrm{C}_{9}$ chain), and in this case, $\mathrm{pp} \approx 0.65$ and so would be more closely associated with that required for planar/vesicle structures. A chain dimension of $\sim 11 \AA$ is also consistent with the bilayer thickness obtained from the modeling, 15 to $24 \AA$. This implies that at the lower concentration there is interdigitation between the two parts of the bilayer, which decreases as the surfactant concentration increases. 
At a surfactant concentration of $7 \mathrm{mM}$ (Figure 2), the scattering data show a different form and has a $Q^{-2}$ dependence. The $Q^{-2}$ dependence implies planar structures, and the lack of any pronounced oscillations implies a structure, consistent with large, flexible unilamellar vesicles .

At higher surfactant concentrations of 10, 15, 20, and $30 \mathrm{mM}$, the microstructure is in yet another form. At lower $Q$ values, the scattering has a $Q^{-4}$ dependence, consistent with interfacial scattering. At higher $Q$ values $\left(Q \approx 0.17 \mathrm{~A}^{-1}\right)$, a relatively broad correlation peak is observed. The relatively broad correlation peak at high $Q$ is not consistent with the much sharper Bragg peak expected from a lamellar phase or multilamellar vesicles. This is supported by optical measurements, where viewed through crossed polarizers the sample shows no observable birefringence, and in a lamellar structure some birefringence would be expected. Hence, the system exhibits short-range order but no long-range order, and there are potentially three solution structures consistent with the light scattering and SANS observations: a sponge phase, a disordered tubule phase, and a disordered vesicle structure.

From the SANS data for the LS sophorolipid at the higher concentrations, the position of the correlation peak is constant and the maximum corresponds to a $Q$ value of approximately $\sim 0.17$ and a $d$ spacing of $\sim 37 \AA$. This value is small compared to the values for typical dilute sponge structures reported in the literature. Hamilton et al. $^{37}$ reported an average value for $d$ of 100-1000 $\AA$, and Porte $^{38}$ reported a value of $\sim 140 \AA$. For sponge structures measured by scattering (neutrons or X-rays), the correlation peak usually appears in the low- to mid- $Q$ region, corresponding to a $d$ spacing of approximately hundreds of angstroms. Hence, it is unlikely that the data here are consistent with the sponge phases that are commonly encountered in dilute solution.

The scattering could be associated with a dilute disordered solution of tubules, where the tubule is formed by wrapping the surfactant bilayer membrane structure in a hollow cylinder. ${ }^{39}$ Tubular structures have been observed in several systems of organic molecules, including phospholipids, ${ }^{40-42}$ glycolipids, ${ }^{43,44}$ and bile salts, ${ }^{45}$ but there is little evidence from scattering measurements on such systems and their origin is the subject of different theoretical approaches. ${ }^{46-48}$

Finally, the scattering could be associated with relatively large, flexible, and highly disordered vesicles, where the disorder and a small number of bilayer shells results in the relatively weak and broad Bragg peaks observed.

Some dynamic light scattering measurements were made, and these results assist in the assignments made on the basis of the birefringence and SANS measurements. The data are shown in Figure 1 in the Supporting Information. At $3 \mathrm{mM}$, the DLS is consistent with predominantly small aggregates, nanovesicles. At $7 \mathrm{mM}$, the DLS is consistent with a very polydisperse bimodal distribution with mean sizes of $\sim 3000 \AA$ and $\sim 2$ to $3 \mu \mathrm{m}$ observed, and the larger component is typical of that obtained from lamellar fragments or large vesicles. At $30 \mathrm{mM}$, the DLS is consistent with aggregates, $\sim 3000 \AA$, but provides no conclusive evidence of the detailed nature of the aggregates, which could be tubules or vesicles.

Acidic Sophorolipids. The self-assembly of two types of acidic sophorolipids (diacetylated and nonacetylated) was investigated using SANS. As discussed previously, it is not possible to obtain AS free from a component of LS. For clarity, the detailed composition information of a range of AS samples is listed in
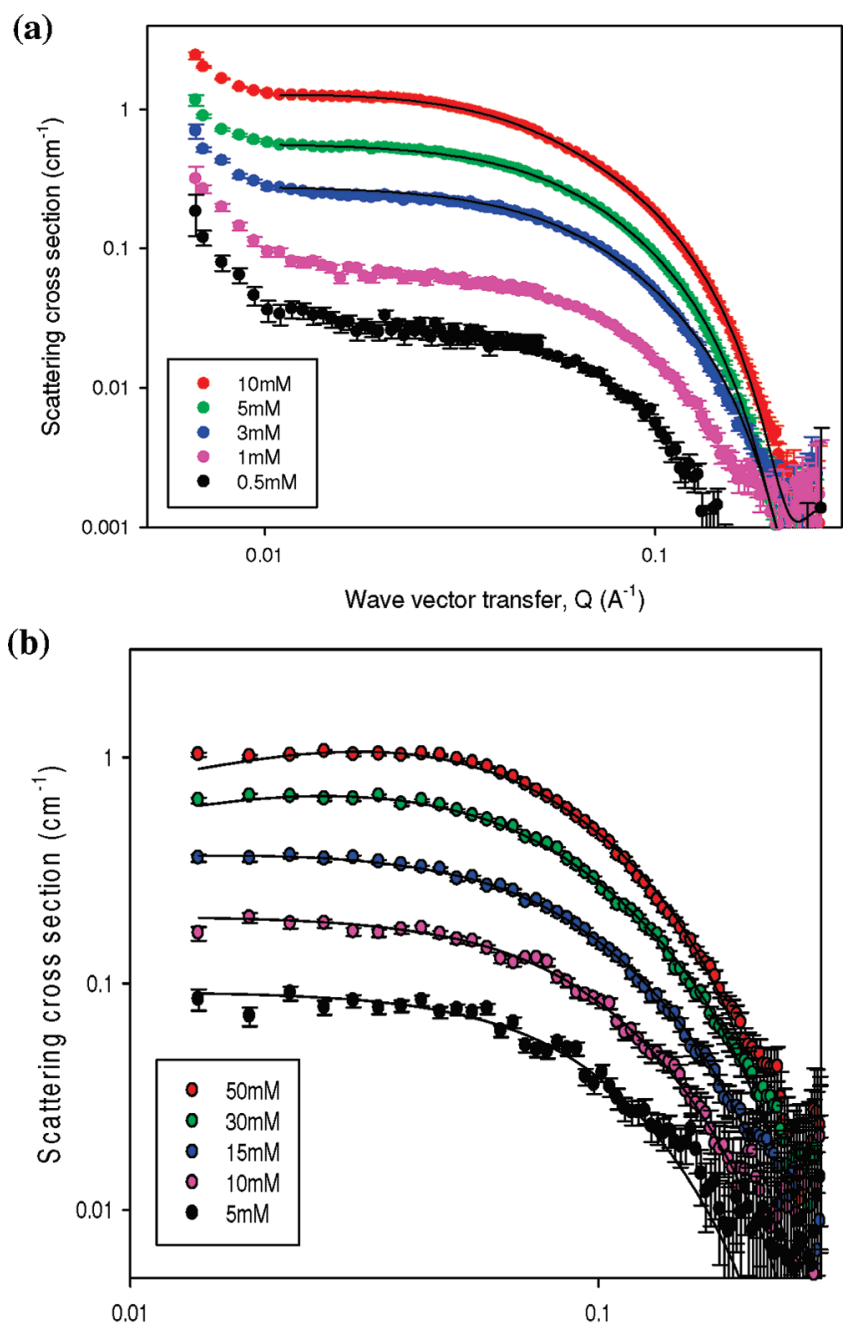

Wave vector transfer, $Q\left(A^{-1}\right)$

Figure 4. SANS data for (a) diacetylated AS (S1) and (b) nonacetylated AS (S4) at different solution concentrations. The solid lines are model calculations as described in the text and for the key model parameters summarized in Table 3.

Table 1, and samples labeled AS(S1) and AS(S4) were used in SANS measurements. Making a comparison between AS(S1) and AS(S4) provides the opportunity to identify the roles of acetylation and the finite LS component in the self-assembly.

An initial visual inspection of the appearance of solutions of diacetylated $\mathrm{AS}(\mathrm{S} 1)$, monoacetylated $\mathrm{AS}(\mathrm{S} 2)$, and nonacetylated sophorolipids (S3) at a solution concentration of $2 \mathrm{mM}$ (above the $\mathrm{cmc}$ ) provides some useful insight into the effect of acetyl groups on the phase structure of sophorolipids. The solutions of AS with one and two acetyl groups had a hazy appearance, indicating the presence of larger aggregates. The solution of AS with no acetyl groups appears to be clear and is consistent with small aggregates such as more highly curved small micellar structures.

For diacetylated acidic sophorolipid AS(S1), SANS measurement were made in the low concentration range from 0.5 to $10 \mathrm{mM}$ (above the $\mathrm{cmc}$ ). Nonacetylated acidic sophorolipid AS(S4) has a much higher water solubility and hence SANS measurements were made over a higher concentration range from 0.5 to $50 \mathrm{mM}$ (above the $\mathrm{cmc}$ ). The SANS data for diacetylated acidic sophorolipid AS(S1) are shown in Figure 4a, where the 
Table 3. Key Model Parameters from Core-Shell Micelle Analysis for Micellar Structures of the Diacetylated and Nonacetylated AS Sophorolipids

\begin{tabular}{|c|c|c|c|c|c|}
\hline effective concentration (mM) & aggregation number, $v$ & surface charge, $z,( \pm 1)$ & $R_{1}(\AA),( \pm 1)$ & $R_{2}(\AA),( \pm 1)$ & ee $( \pm 0.1)$ \\
\hline 3 mM AS(S1) (2-acetyl 8\% LS) & $85 \pm 4$ & 0 & 15 & 20 & 2.7 \\
\hline 5 mM AS(S1) (2-acetyl 8\% LS) & $97 \pm 4$ & 0.5 & 16 & 21 & 2.7 \\
\hline 10 mM AS(S1) (2-acetyl 8\% LS) & $116 \pm 4$ & 1.6 & 15 & 21 & 3.4 \\
\hline 5 mM AS (S4) (0-acetyl) & $28 \pm 2$ & 0.1 & 11 & 14 & 2.4 \\
\hline 10 mM AS(S4) (0-acetyl) & $30 \pm 2$ & 0.1 & 11 & 13 & 2.7 \\
\hline 15 mM AS(S4) (0-acetyl) & $32 \pm 2$ & 0.1 & 12 & 15 & 2.9 \\
\hline 30 mM AS (S4) (0-acetyl) & $37 \pm 2$ & 0.1 & 12 & 15 & 2.7 \\
\hline 50 mM AS (S4) (0-acetyl) & $40 \pm 2$ & 1.6 & 11 & 14 & 3.2 \\
\hline
\end{tabular}

micellar component is fitted using the core-shell micelle model, ${ }^{21}$ as illustrated in the Figure. The SANS data for nonacetylated acidic sophorolipid AS(S4) are shown in Figure 4b and analyzed using the same micelle model. The key model parameters obtained from data analysis are summarized in Table 3.

For AS(S1) (diacetylated AS with 8.4\% LS), the scattering is predominantly from globular micelles, $\mathrm{L}_{1}$, but in a mixed phase $\left(\mathrm{L}_{1} / \mathrm{L}_{\alpha}\right.$, where $\mathrm{L}_{1}$ is the predominant phase) over the concentration range measured. The upturn in the scattering at low $Q$ is consistent with the coexistence of a much smaller fraction of larger structures, and this minor component is assumed to be a contribution from a coexisting lamellar component. For AS(S4) (nonacetylated AS without LS), the scattering is consistent with small micellar structures at all of the surfactant concentrations measured and without any evidence of a lamellar contribution visible. The $\mathrm{AS}(\mathrm{S} 1)$ micelles have a much higher aggregation number and are larger than the AS(S4) micelles. The two extra acetyl groups make the AS more hydrophobic than AS(S4). However, because the S1 sample also contains $8.4 \%$ LS, which itself has a strong tendency to form more planar structures, both factors contribute to the increase in the aggregate size. A common feature of both AS(S4) and AS(S4) micelles is that all of the micelles are very weakly charged at all concentrations, confirming that AS is weakly ionic like the LS component.

The Israelachvili, Mitchell, and Ninham packing parameter ${ }^{36}$ (PP) has been effective in predicting the general trends in the evolution of surfactant self-assembled structures, as discussed earlier. For AS, taking the known values of molecular volumes of hydrophobic chain $V$ of $512 \AA^{3}$, the fully extended chain length $l$ of $24 \AA$, and the area per molecule $A$ from surface tension or neutron reflectivity data, the PP values for diacetyled AS(S1) and nonacetylated AS(S4) are 0.22 and 0.20 , respectively. For nonacetylated AS(S4), the PP value is consistent with the formation of small micelles, $\mathrm{L}_{1}$, which is in good agreement with the SANS data. For diacetylated AS(S1), the PP value also indicates the formation of small micelles, $\mathrm{L}_{1}$, but does not readily account for the $\mathrm{L}_{1} / \mathrm{L}_{\alpha}$ mixed-phase behavior.

The micelle SANS data are modeled using a core-shell model where the inner core contains the alkyl chains and the outer shell contains the headgroups and associated hydration. Previously, ${ }^{24}$ the inner core radius has been constrained to be $l$, the fully extended alkyl chain length, and elliptical growth is assumed for aggregation numbers greater than that required to space fill the volume defined by a radius $l$. As discussed earlier, the alkyl chain geometry of the AS and especially the LS components introduces some uncertainty into what value the inner radius, $R_{1}$, should be constrained to. Hence, in the modeling used here that constraint
Table 4. Key Model Parameters for the Core-Shell Micelle Analysis of an LS/AS(S1) Mixture at Different Concentrations from 5 to $15 \mathrm{mM}$ and at a Fixed Composition of 40/60

\begin{tabular}{ccccc}
$\begin{array}{c}\text { concentration } \\
(\mathrm{mM})\end{array}$ & $\begin{array}{c}R_{1} \\
\text { aggregation } \\
\text { number, } v\end{array}$ & $\begin{array}{c}R_{2} \\
( \pm 1),\end{array}$ & $\begin{array}{c}(\AA), \\
( \pm 1)\end{array}$ & $\begin{array}{c}\text { ee } \\
( \pm 0.1)\end{array}$ \\
5 & $53 \pm 2$ & 12 & 15 & 3.5 \\
10 & $73 \pm 4$ & 12 & 15 & 5.2 \\
15 & $103 \pm 6$ & 11 & 14 & 9.2 \\
\hline
\end{tabular}

has been relaxed and $R_{1}$ and ee (the elliptical axial ratio) both become refinable parameters while retaining the space-filling criterion.

The parameters in Table 4 show that for both AS (S1 and S4) samples $R_{1}$ is significantly less than the fully extended chain length of a $\mathrm{C}_{18}$ chain $(\sim 24 \AA)$. As discussed earlier, this would not be surprising for the LS sophorolipid with its lactone ring structure, and it suggests that the double bond in the AS chain results in a highly bent alkyl chain conformation for the AS alkyl chains in the micellar structure. The $R_{1}$ values for AS (S1) and AS (S4) are 15 and $12 \AA$, respectively, $\sim 65$ and $50 \%$ of the fully extended chain length. This implies that the chain structure and the associated packing constraints result in a highly bent conformation within the micelle.

Although the relative sizes of the micelles for the acetylated and nonacetylated AS samples can be explained in terms of the greater hydrophobicity of the diacetylated AS and the presence of $8 \% \mathrm{LS}$, there are some changes in the micelle radius for which the origin is less obvious. The radius of diacteylated AS(S1) (with $8 \%$ LS) is $\sim 15 \AA$, compared to $\sim 12 \AA$ for nonacteylated AS(S4). This implies that the acetylation of the headgroup and its increased hydrophobicity and the packing constraints associated with the small amount of LS present result in a slightly larger hydrophobic core. As observed later, this is not sustained over the entire AS/LS composition range, where $R_{1}$ is predominantly $\sim 12 \AA$, and we will return to this point later in the discussion.

Surfactant Mixtures. LS/AS Mixtures. Figure 5 shows some typical SANS data and the associated model fits for the LS/AS (S1) mixtures at a fixed solution composition of 40/60 and at concentrations of $5,10,15$, and $30 \mathrm{mM}$.

The SANS data in Figure 5 illustrate a typical variation in the scattering for the LS/AS (S1) mixture with the increase in solution concentration. For surfactant mixtures with concentrations of up to $15 \mathrm{mM}$, the scattering data are consistent with small globular noninteracting micelles. The scattering was modeled using the micellar core-shell model, ${ }^{21}$ and the key model parameters are 


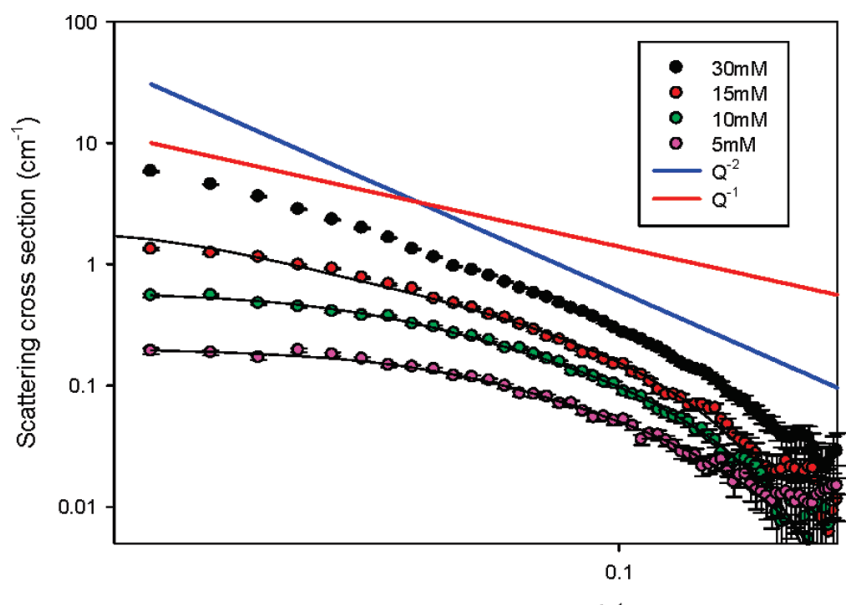

Wave vector transfer, $Q\left(\AA^{-1}\right)$

Figure 5. SANS data for the binary mixture of LS/AS (S1) at a fixed composition of $40 / 60$ and at different concentrations. The solid lines are model fits using the parameters in Table 5. The straight lines illustrate $Q^{-1}$ and $Q^{-2}$ scattering dependences.

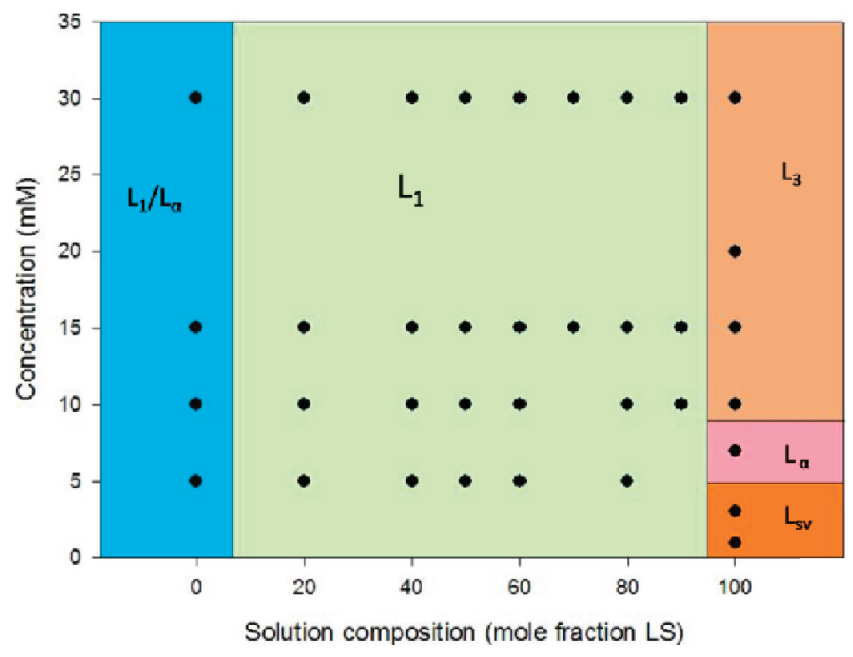

Figure 6. Phase diagram for the LS/AS (S1) mixture.

listed in Table 4. At the higher solution concentration of $30 \mathrm{mM}$, the scattering data are no longer consistent with small globular micelles. In the low- $Q$ region, the data tend toward a $Q^{-1}$ dependence, consistent with the formation of more highly elongated rodlike structures.

With an increase in surfactant concentration, the aggregation number of mixed micelles increases and the micelle ellipticity increases, consistent with the micelles becoming more elongated. The surface charge is insignificant across the whole range of concentration measured and implies that the micelle surface is only very weakly charged. This is consistent with the individual LS and AS components being weakly charged, as discussed previously.

The SANS data presented in Figure 2 in the Supporting Information, measured at a fixed surfactant concentration of $15 \mathrm{mM}$ and at different compositions, are typical of the LS/AS (S1) mixtures. The data are also fitted to the globular micelle core-shell model, and the model parameters are listed in Table 1 in the Supporting Information. As the surfactant mixture becomes richer in the AS component, the aggregation number of

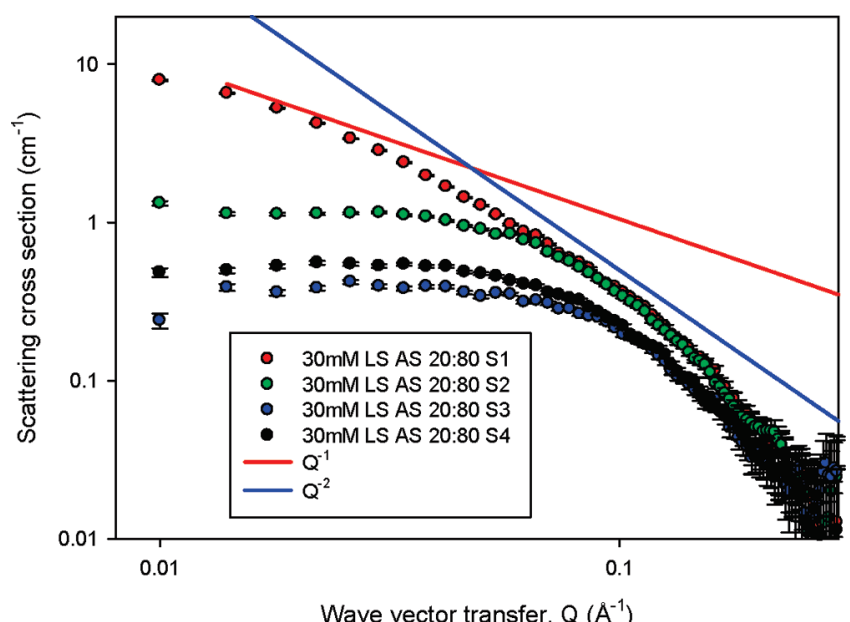

Figure 7. SANS data for the binary mixture of LS/AS (S1, S2, S3, and S4) at a fixed composition of $20 / 80$ and at a concentration of $30 \mathrm{mM}$. The solid lines illustrate $Q^{-1}$ and $Q^{-2}$ scattering dependencies.

the micelles increases and the micelles also become more elliptical. The surface charge is again negligible across the whole range of composition measured.

From these data, it is possible to construct a phase diagram of LS/AS (S1), and this is shown in Figure 6.

In the LS/AS (S1) phase diagram, over the majority of the concentration and composition parameter space explored, the self-assembly is dominated by the formation of small globular micelles, $\mathrm{L}_{1}$, in the composition range from 20/80 LS/AS (S1) to 90/10 LS/AS (S1). Although the data for AS(S1) (Figure 4) indicated only $\mathrm{L}_{1} / \mathrm{L}_{\alpha}$ coexistence, all of the subsequent measurements (apart from LS only) indicated a purely $\mathrm{L}_{1}$ phase. It has been shown previously that AS (S1) forms $\mathrm{L}_{1} / \mathrm{L}_{\alpha}$ structures on its own whereas LS adopts a morphology with a lower curvature, forming nanovesicle structures at lower concentrations (<7 mM) and a disordered tubule structure at higher concentrations. Hence, in the LS/AS(S1) mixtures the AS(S1) component dominates the self-assembly, and this is in contrast to the adsorption behavior at the air-water interface where the greater surface activity of the LS component dominates the adsorption.

In earlier measurements, it was shown that the level of acetylation and a small fraction of the LS component had an impact on the self-assembly. We have investigated the effect of acetylation on the self-assembly behavior of sophorolipids at a fixed AS/LS composition where the degree of acetylation was the only variable. Measurements were made for a surfactant mixture of LS/AS for the different AS isomers (AS(S1), diacetylated; AS(S2), monoacetylated; AS(S3), nonacetylated; and AS(S4), nonacetylated) at a fixed composition of 20:80 and at surfactant concentrations of 15 and $30 \mathrm{mM}$. Figures 7 and 3 in the Supporting Information show the corresponding scattering data.

All of the scattering data shown in Figures 7 and 3 in the Supporting Information are consistent with noninteracting globular micelles. The scattering data for LS/AS (S1) at a solution concentration of $30 \mathrm{mM}$ show a $Q^{-1}$ dependence, indicating the formation of more highly elongated structures. Apart from the scattering data at the higher surfactant concentration, the other AS samples with different degrees of acetylation (S1-S4) are consistent with the formation of relatively small globular micelles, which show mostly only modest variations in size. The data were 


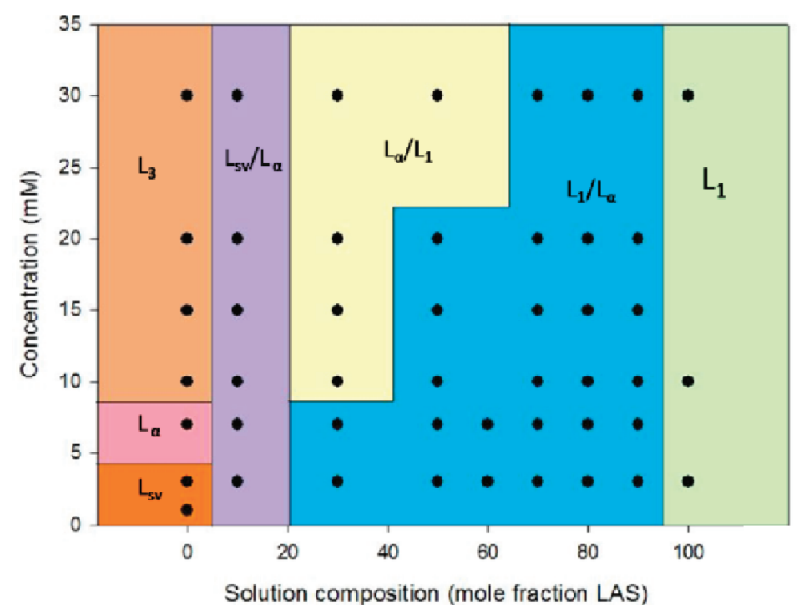

Figure 8. Phase diagram for the LS/LAS mixture.

modeled using the core-shell micelle model, ${ }^{21}$ and the key model parameters are summarized in Table 2 in the Supporting Information.

With a decreasing number of acetyl groups from S1 (diacetylated) to S3 (nonacetylated), the aggregation number and the ellipticity of the resultant micelles decreases at both 30 and $15 \mathrm{mM}$ solution concentrations. This is consistent with the surfactant mixture that contains diacetylated AS(S1) forming larger and more elongated structures than mixtures containing monoacetylated or nonacetylated AS(S4), and this implies that the presence of acetyl groups increases the hydrophobicity of the surfactant and reduces the preferred curvature of aggregates, as was inferred earlier.

Otto et al. ${ }^{12}$ reported that the diacetylated AS was more surface-active than the monoacetylated AS, and this is consistent with the difference in the overall hydrophobicity of the molecule. Unlike conventional surfactants, where the molecular structure can be divided into two clear domains of hydrophobic (chain) and hydrophilic (headgroup) moieties, the hydrophobic acetyl groups of the sophorolipids are attached to the hydrophilic sophorose sugar headgroup, and for the LS molecule, both ends of the fatty acid chain are attached to the sophorose sugar headgroup in a highly bent configuration. It is thought that this unusual structure feature is responsible for the formation of the compact monolayer described above and the relatively elongated structures that are formed here, as was discussed earlier.

LS/LAS Mixtures. From a series of SANS measurements for LS/LAS mixtures, it has been possible to construct a phase diagram for LS/LAS, and this is shown in Figure 8.

At the higher surfactant concentrations and for surfactant mixtures rich in LS, predominantly planar structures are formed. Over much of the phase diagram, there is a large region of $\mathrm{L}_{1}$ and $\mathrm{L}_{\alpha}$ coexistance that can be subdivided into two distinct regions, one where $\mathrm{L}_{\alpha}$ dominates and the other where $\mathrm{L}_{1}$ dominates. For pure LAS, highly curved micellar structures, $\mathrm{L}_{1}$, are formed at these relatively low surfactant concentrations.

In Figure 9, the scattering data for the LS/LAS mixture at a fixed composition of $90 / 10$ and at different concentrations of 3 , $7,10,15,20$, and $30 \mathrm{mM}$ are presented.

At all of the concentrations measured at this composition, the scattering data are consistent with an $\mathrm{L}_{\mathrm{sv}} / \mathrm{L}_{\alpha}$ mixed phase, with the small vesicle, $\mathrm{L}_{\mathrm{sv}}$, component making the dominant contribution to the scattering. In the low- $Q$ region, the upturn in the

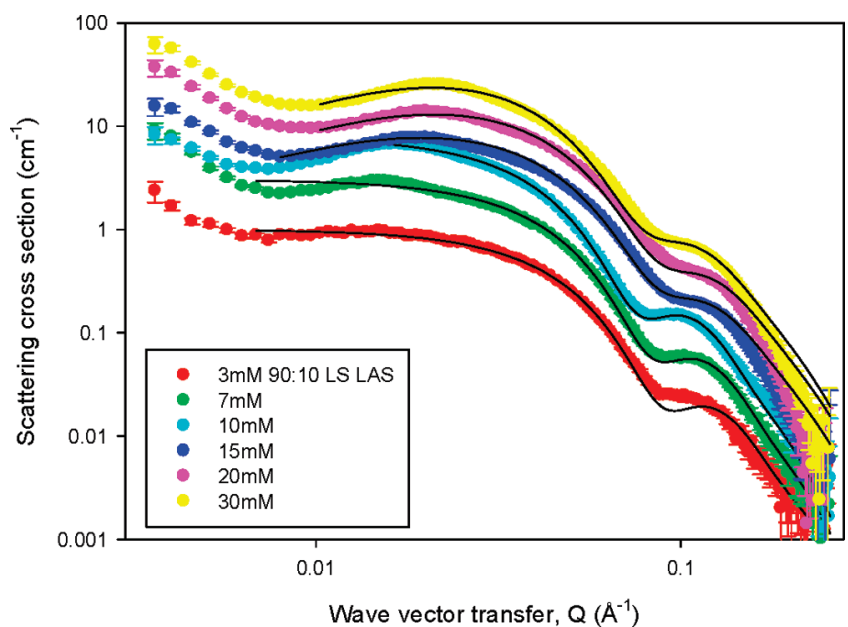

Figure 9. SANS data for the binary mixture of LS/LAS at a fixed composition of $90 / 10$ and at different concentrations (the plots are shifted vertically for clarity). The solid lines are model calculations as described in the text and for parameters in Table 5.

Table 5. Key Model Parameters for Core-Shell Micelle Analysis for Small Vesicle Structures by LS/LAS Mixtures at a Fixed Composition of 90/10 and at Different Concentrations

\begin{tabular}{ccccc}
$\begin{array}{c}\text { concentration } \\
(\mathrm{mM})\end{array}$ & $\begin{array}{c}R_{1} / \AA \\
( \pm 2)\end{array}$ & $\begin{array}{c}R_{2} / \AA \\
( \pm 2)\end{array}$ & $\begin{array}{c}\text { polydispersity, } \\
\sigma( \pm 0.05)\end{array}$ & $\begin{array}{c}\text { charge, } \\
3\end{array}$ \\
22 & 38 & 0.2 & $1 \pm 1$ \\
7 & 23 & 41 & 0.2 & 1 \\
10 & 25 & 46 & 0.2 & 1 \\
15 & 20 & 36 & 0.25 & $5 \pm 3$ \\
20 & 20 & 36 & 0.25 & 5 \\
30 & 22 & 40 & 0.025 & 7 \\
\hline
\end{tabular}

scattering has a $Q^{-2}$ dependence, consistent with planar structures and unilamellar vesicles. At an intermediate $Q$ value, as the surfactant concentration increases from 3 to $30 \mathrm{mM}$, the broad peak in the scattering pattern becomes more pronounced. This is due to the increased repulsive interaction between the nanovesicles as the concentration increases. In the region of relatively higher $Q$ values, the distinctive shallow minimum is the signature of the formation of small vesicles $L_{s v}$, and this is similar to pure $L S$ data at concentrations of up to $7 \mathrm{mM}$, as discussed previously. The SANS data at a concentration of 3 to $30 \mathrm{mM}$ are modeled by the small vesicle model as described previously, and the model parameters are summarized in Table 5 .

The size of the nanovesicles goes through a weak maximum at a surfactant concentration of $\sim 10 \mathrm{mM}$. At the higher surfactant concentrations, there is an increasing contribution from the $\mathrm{L}_{\alpha}$ or large vesicle component to the scattering. With increasing concentration, the internanovesicle interaction peak becomes more pronounced. This is in part due to the increased concentration (and hence the volume fraction contribution to the interaction) but also due to an increase in the surface charge. The surface charge arises from the LAS contribution, but because the measurements were made at a fixed composition, this increase would imply a change in the distribution of the LS and LAS components between the two competing microstructures. This would also be consistent with the change in the size of 


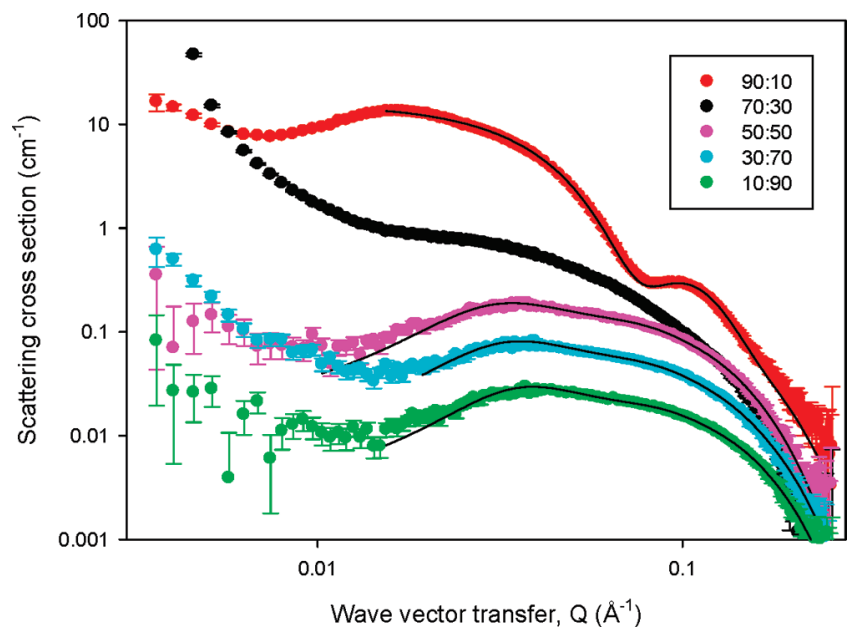

Figure 10. SANS data for the binary mixture of LS/LAS at a fixed concentration of $10 \mathrm{mM}$ and at different compositions. (The plots are shifted vertically for clarity.) The solid lines are model calculations for globular micelles for the parameters in Table 6.

Table 6. Key Model Parameters from Core-Shell Micelle Analysis for LS/LAS Mixture at Fixed Concentration of $10 \mathrm{mM}$ and at Different Compositions

\begin{tabular}{cccccc} 
composition aggregation & surface charge, $z$, & $R_{1}(\AA)$, & $R_{2}(\AA)$, & ee \\
LS/LAS & number, $v$ & $( \pm 1)$ & $( \pm 1)$ & $( \pm 1)$ & $( \pm 0.1)$ \\
$50 / 50$ & $74 \pm 4$ & 13 & 16 & 18 & 1.8 \\
$30 / 70$ & $60 \pm 3$ & 14 & 15 & 17 & 1.8 \\
$10 / 90$ & $47 \pm 2$ & 13 & 14 & 16 & 1.8 \\
\hline
\end{tabular}

the nanovesicles to smaller, more highly curved nanovesicles as the charge increases. In principle, this change could be measured by $\mathrm{H} / \mathrm{D}$ isotopic substitution, but probably not with sufficient accuracy to determine the relatively subtle changes that would be required to show the effect. The quality of the model fits in the intermediate $Q$ region, where the contribution from $S(Q)$ and hence the surface charge is important, shows some variability. This is a result of the uncertainty associated with the contribution from the scattering from the minority $\mathrm{L}_{\alpha}$ component. The uncertainty in the surface charge values in Table 5 reflect this, but the qualitative increase with increasing concentration is nevertheless evident.

Figure 10 shows the scattering data at a fixed LS/LAS concentration of $10 \mathrm{mM}$ and at different surfactant compositions.

For surfactant mixtures rich in LAS (composition from 10:90 to 50:50 LS/LAS), the scattering profiles are consistent with $\mathrm{L}_{1} / \mathrm{L}_{\alpha}$ coexistence but with the $\mathrm{L}_{1}$ component contributing predominantly to the scattering. For the mixtures at these compositions, the broad peak in the mid- $Q$ region indicates repulsive micellar interaction arising from the micellar charge due to the LAS component. These SANS data are fitted to the micellar core-shell model, and the key model parameters are listed in Table 6 .

For surfactant mixtures that are relatively rich in LS (50/50) to the mixtures rich in LAS, the aggregation number decreases from 74 at LS/LAS 50/50 to 47 at 10/90. At these concentrations, the pure LS tends to form larger planar structures whereas the pure LAS tends to form smaller globular micelles. The trends exhibited in these mixtures are consistent with the competition

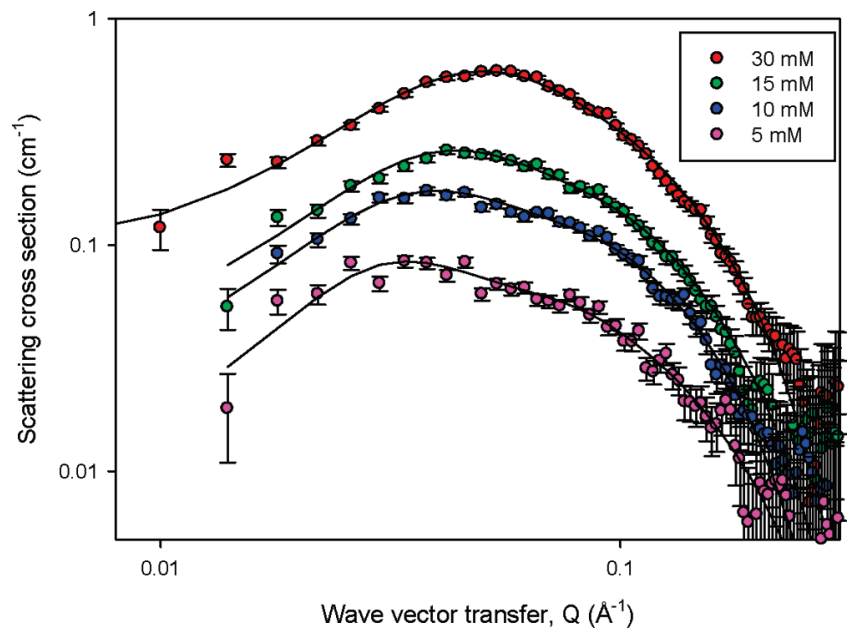

Figure 11. SANS data for AS(S4)/LAS mixtures at fixed composition (60:40) and for different surfactant concentrations. The solid lines are model calculations from the core-shell micelle model using the parameters in Table 3 in the Supporting Information.

between the preferred curvatures and the structures of each individual component such that at $70 / 30$ there is $\mathrm{L}_{\alpha} / \mathrm{L}_{1}$ coexistence and at $90 / 10$ there is $\mathrm{L}_{\mathrm{sv}} / \mathrm{L}_{\alpha}$ coexistence.

AS (S4, Nonacetylated)/LAS Mixtures. Figure 11 shows the typical SANS data for AS(S4)/LAS mixtures. The data are consistent with small globular micelles, and the broad peak at intermediate $Q$ values arises from repulsive intermicellar interactions.

The pure AS (0-acetyl) and the pure LAS both form small globular micelles $\left(\mathrm{L}_{1}\right)$, and hence it is not surprising that the AS(S4)/LAS mixtures of different compositions form an $\mathrm{L}_{1}$ phase. The SANS data have been modeled using the interacting micellar core-shell model, and the key model parameters are listed in Table 3 in the Supporting Information. The variations in the aggregation number and the degree of ionization of mixed micelles are shown in Figure 4 in the Supporting Information. As shown in Figure 4 in the Supporting Information, the micellar aggregation number increases modestly with surfactant concentration but is within error invariant with solution composition. The other notable trends shown in Figure 4 in the Supporting Information are that the degree of ionization of the micelles, expressed as $\delta={ }^{z} / \gamma$, decreases with the increase in surfactant concentration and increases with the increase in LAS concentration. A similar trend was described previously for binary surfactant mixtures of $R_{2} /$ LAS. $^{10}$ The increase in the micelle charge with increasing LAS composition is not entirely unexpected because the sophorolipid is only weakly ionic and LAS is strongly ionic. However, the charge does not increase linearly with composition but saturates at a solution composition of $\sim 50$ to $60 \mathrm{~mol} \%$ LAS. Furthermore, the charge decreases with increasing surfactant concentration. Both effects are linked and are related to a self-screening effect of the neighboring micelles, which is more significant for the higher LAS compositions and higher surfactant (and hence micelle) concentrations.

As previously discussed, for purely ionic surfactants, $\delta$ is typically between 0.25 and $0.3 .^{24}$ Lower values of $\delta$ and their variations with solution composition for SDS/nonionic surfactant mixtures were reported by Penfold et al. ${ }^{49}$ In that study, values of $\delta$ $\leq 0.1$ were reported for SDS $/ \mathrm{C}_{12} \mathrm{E}_{6}$ and $\mathrm{SDS} / \mathrm{C}_{12} \mathrm{E}_{8}$ mixtures that were rich in the nonionic surfactant ( $>80 \mathrm{~mol} \%$ nonionic). 
However, for solutions $>50 / 50 \mathrm{~mol}$ ratio of SDS/nonionic mixtures the micelle surface charge was similar to that for pure SDS micelles. The AS(S4)/LAS micelles are much more highly charged, especially at lower surfactant concentrations. The absolute values of $v$ and $\delta$ are consistent with those previously reported for rhamnolipid/LAS mixtures, ${ }^{10}$ for LAS/nonionic mixtures, ${ }^{17}$ and for SDS/nonionic mixtures. ${ }^{49}$ The general trends observed for $\delta$ with surfactant concentration are consistent with those reported by Hayter and Penfold ${ }^{24}$ and elsewhere. ${ }^{49}$ This trend was considered in more detail by Hayter ${ }^{50}$ in the context of the "dressed micelle" model of Evans, Mitchell, and Ninham. ${ }^{51}$ An analytical expression was derived for the excess ion adsorption onto the micelle surface and provided good agreement with experimental data $^{24}$ and describes the observed decrease in $\delta$ with increasing surfactant concentration.

Ternary LS/AS(S1)/LAS Surfactant Mixtures. Figure 5 in the Supporting Information shows some typical SANS data for the ternary mixture of LS AS(S1) (1:1)/LAS at a composition of $60 / 40$ and at different concentrations. Similar to AS/LAS mixtures, all of the scattering data for the ternary mixtures are consistent with interacting small globular micelles. The broad peak in the intermediate $Q$ region indicates a relatively strong repulsive micellar interaction at higher surfactant concentrations. The data shown in Figure 5 in the Supporting Information are typical of all of the SANS data for the ternary surfactant mixtures. That is, in all three ternary systems with different LS/AS(S1) ratios $(2: 1,1: 1$, and $1: 2)$, only small interacting globular micelles are formed. It has been possible to analyze the SANS data using the interacting micellar core-shell model, and the micelle aggregation numbers from the model fitting are listed in Table 4 in the Supporting Information. Because of the similarities to the analysis of the AS(41)/LAS mixtures, a more detailed description of the model parameters is not included.

Similar to the AS(S4)/LAS binary mixtures, the aggregation numbers for the three ternary systems are broadly similar at the different compositions and show a modest increase with surfactant concentration. The aggregation number is relatively small, the micelles are globular, and the micelle surface charge increases modestly with increasing LAS content and decreases with increasing surfactant concentration, as observed in the AS(S4)/LAS binary mixtures. In the binary mixtures of LS/AS(S4), the major region of the phase diagram is in the form of $L_{1}$. However, for the LS/LAS mixture, a more complicated phase evolution is observed, from planar structures formed by mixtures rich in $\mathrm{LS}$ to an $\mathrm{L}_{1}$-dominant $\mathrm{L}_{1} / \mathrm{L}_{\alpha}$ mixed phase formed by $\mathrm{LAS}$ rich compositions.

In all three ternary mixture systems, only the $\mathrm{L}_{1}$ phase is formed. It is therefore clear that $\mathrm{AS}(\mathrm{S} 1)$ dominates the phase behavior, resulting in higher curvature structures being formed by the surfactant mixtures of different compositions. This trend is in marked contrast to the surface behavior discussed earlier in conjunction with the neutron reflectivity results. In that case, the LS component dominates the surface behavior for all three ternary mixture systems. The particular structures of the LS and AS sophorolipid components therefore result in different behavior at the surface and in solution.

\section{SUMMARY AND CONCLUSIONS}

The unusual molecular structure associated with the lactone ring of the LS sophorolipid component results in an unusual evolution in its self-assembly in aqueous solution. Measurements were made at relatively low surfactant concentrations because the hydrophobicity of the LS component limits its solubility. Furthermore, because the LS sophorolipid is only weakly ionic the resulting aggregates are only weakly charged. The packing constraints associated with the lactone ring structure of the alkyl chains resulted in the formation of small unilamellar vesicles, nanovesicles, at low surfactant concentrations. At higher concentrations, there was a transformation via a large, flexible unilamellar vesicle phase to a disordered lamellar phase, and there was no evidence of a simple globular micellar phase.

In contrast, the AS sophorolipid component forms small globular micelles over a wide concentration range. However, the double bond in the alkyl chain results in a highly bent chain conformation in the micelles, and the micelle sizes reflect this constraint. The degree of acetylation of the headgroup of the AS component affects the degree of hydrophobicity and the micelle size.

In AS/LS mixtures, the micellar structure of the AS component dominates, and apart from the LS-rich compositions, the resulting mixed surfactant microstructure is predominantly small globular micelles. The addition of the LS component acts in a similar way to the increased acetylation of the AS component and promotes modest micellar growth. Mixtures of AS with anionic surfactant LAS and the LS/AS/LAS ternary mixtures are dominated by the preference for the higher preferred curvature of the AS and LAS components and form small globular micelles over most of the composition range studied.

The unusual molecular structure of the LS sophorolipid component also has an impact upon the structure of the mixed LS/LAS aggregates. For LS-rich compositions, the region of occurrence of the nanovesicles is extended. Micellar/lamellar coexistence occurs over much of the composition range, and the solutions rich in LAS are predominantly micellar. The contrasting behaviors of the LS component with the AS component and LAS suggest that the headgroup geometry plays an important role in determing the mixed microstructure in addition to the role of the alkyl chain structure.

\section{ASSOCIATED CONTENT}

S Supporting Information. Additional SANS data plots, model parameters, and DLS data plots. This material is available free of charge via the Internet at http://pubs.acs.org.

\section{AUTHOR INFORMATION}

\section{Corresponding Author}

*E-mail: jeff.penfold@stfc.ac.uk.

\section{ACKNOWLEDGMENT}

We acknowledge the provision of neutron beam time at ISIS (LOQ, SANS2D) and at the ILL (D11, D22) and the invaluable assistance of the Instrument Scientists at ISIS and the ILL. The support of the project by Unilever and the dti Technology Programme is acknowledged.

\section{REFERENCES}

(1) Holmberg, K.; Kronberg, B. J.; Lindman, B. Surfactants and Polymers in Aqueous Solution, 2nd ed.; John Wiley \& Sons: Hoboken, NJ, 2002. 
(2) Lang, S.; Wagner, F. In Biosurfactants: Production, Properties, Applications; Kosaric, N., Ed.; Marcel Dekker: New York, 1993.

(3) Zajic, J. E.; Gerson, H. D. G. F. Biotechnol. Bioeng. 1977, 19, 1303-1320.

(4) Georgiou, G. L.; Lin, S.-C.; Sharma, M. M. Nat. Biotechnol. 1992, $10,60-65$.

(5) Kretschmer, A.; Bock, H.; Wagner, F. Appl. Environ. Microbiol. 1982, 44, 864-870.

(6) Koenig, S.; Quitzsch, K.; Koenig, B.; Hommel, R.; Haferburg, D.; Kleber, H. P. Colloids Surf., B 1993, 1, 33-41.

(7) Zhu, Y.; Gan, J. J.; Zhang, G. L.; Yao, B.; Zhu, W. J.; Meng, Q. J. Zhejiang Univ., Sci., A 2007, 8, 1514-1520.

(8) Rosenberg, E.; Ron, E. Z. Appl. Microbiol. Biotechnol. 1999, $52,154-162$.

(9) Chen, M.; Penfold, J.; Thomas, R. K.; Smyth, T. J. P.; Perfumo, A.; Marchant, R.; Banat, I.; Stevenson, P.; Parry, A.; Tucker, I. Langmuir 2010, 26, 18281-18292.

(10) Chen, M.; Penfold, J.; Thomas, R. K.; Smyth, T. J. P.; Perfumo, A.; Marchant, R.; Banat, I.; Stevenson, P.; Parry, A.; Tucker, I. Langmuir 2010, 26, 17958-17968.

(11) Chen, M.; Penfold, J.; Thomas, R. K.; Smyth, T. J. P.; Perfumo, A.; Marchant, R.; Banat, I.; Stevenson, P.; Parry, A.; Tucker, I. Langmuir 2011 submitted for publication.

(12) Van Bogaert, I. N. A.; Saerens, K.; De Muynck, C.; Develter, D.; Soetaert, W.; Vandamme, E. J. Appl. Microbiol. Biotechnol. 2007, 76, 23-34.

(13) Zhou, S. Q.; Xu, C.; Wang, J.; Gao, W.; Akhverdiyeva, R.; Shah, V.; Gross, R. Langmuir 2004, 20, 7926-7932.

(14) Zhang, L.; Somasundaran, P.; Singh, S. K.; Felse, A. P.; Gross, R. Colloids Surf., A 2004, 240, 75-82.

(15) Otto, R. T.; Daniel, H. J.; Pekin, G.; Muller-Decker, K.; Furstenberger, G.; Reuss, M.; Syldatk, C. Appl. Microbiol. Biotechnol. 1999, 52, 495-501.

(16) Smyth, T. J. P., A.; Marchant, R.; Banat, I. M.; Chen, M.; Thomas, R. K.; Penfold, J.; Stevenson, P. S.; Parry, N. J. Appl. Microbiol. Biotechnol. 2010, 87, 1347-1354.

(17) Penfold, J.; Thomas, R. K.; Dong, C. C.; Tucker, I.; Metcalfe, K.; Golding, S.; Grillo, I. Langmuir 2007, 23, 10140-10149.

(18) D11 diffractometer at ILL. http://www.ill.eu/instrumentssupport/instruments-groups/instruments/d11/.

(19) D22 diffractometer at ILL. http://www.ill.eu/d22/.

(20) http://www.isis.stfc.ac.uk/instruments/sans2d/sans2d3000. html.

(21) Heenan, R. K.; King, S. M.; Penfold, J. J. Appl. Crystallogr. 1997, 30, 1140 .

(22) Ghosh, R. E.; Egelhaaf, S. U.; Rennie, A. R. ILL Internal Report, ILL-98GH14T, 1998.

(23) Heenan, R. K.; King, S. M.; Osborn, R.; Stanley, H. B. RAL Internal Report, RAL-89-128, 1989.

(24) Hayter, J. B.; Penfold, J. Colloid Polym. Sci. 1983, 261, 1022-1030.

(25) Hayter, J. B.; Penfold., J. Mol. Phys. 1981, 42, 109-118.

(26) Provencher, S. W. Makromol. Chem. 1979, 180, 201.

(27) Coldren, B. A.; Warriner, H.; van Zanten, R.; Zasadzinski, J. A. Langmuir 2006, 22, 2465-2473.

(28) Gonzalez, Y. I.; Stjerndahl, M.; Danino, D.; Kaler, E. W. Langmuir 2004, 20, 7053-7063.

(29) Tucker, I.; Penfold, J.; Thomas, R. K.; Bradbury, R.; Grillo, I. Langmuir 2009, 25, 4934-4944.

(30) Grillo, I.; Penfold, J.; Tucker, I.; Cousin, F. Langmuir 2009, $25,3932-3943$.

(31) Oberdisse, J.; Couve, C.; Appell, J.; Berret, J. F.; Ligoure, C.; Porte, G. Langmuir 1996, 12, 1212-1218.

(32) Oberdisse, J.; Porte, G. Phys. Rev. E 1997, 56, 1965-1975.

(33) Oberdisse, J. Eur. Phys. J. B 1998, 3, 463-469.

(34) Jung, H. T.; Coldren, B.; Zasadzinski, J. A.; Iampietro, D. J.; Kaler, E. W. Proc. Natl. Acad. Sci. U.S.A. 2001, 98, 1353-1357.

(35) Jung, H. T.; Lee, S. Y.; Kaler, E. W.; Coldren, B.; Zasadzinski, J. A. Proc. Natl. Acad. Sci. U.S.A. 2002, 99, 15318-15322.
(36) Israelachvili, J. N.; Mitchell, D. J.; Ninham, B. W. J. Chem. Soc., Faraday Trans. 1976, 72, 1525-1568.

(37) Hamilton, W. A.; Porcar, L.; Butler, P. D.; Warr, G. G. J. Chem. Phys. 2002, 116, 8533-8546.

(38) Porte, G.; Appell, J.; Bassereau, P.; Marignan, J.; Skouri, M.; Billard, I.; Delsanti, M. Physica A 1991, 176, 168-186.

(39) von Berlepsch, H.; Bottcher, C.; Quart, A.; Regenbrecht, M.; Akari, S.; Keiderling, U.; Schnablegger, H.; Dahne, S.; Kirsten, S. Langmuir 2000, 16, 5908.

(40) Chiruvolu, S.; Warriner, H. E.; Naranjo, E.; Idziak, S. H. J.; Radler, J. O.; Plano, R. J.; Zasadzinski, J. A.; Safinya, C. R. Science 1994, 266, 1222-1225.

(41) Mishra, B. K.; Thomas, B. N. J. Am. Chem. Soc. 2002, 124, 6866-6871.

(42) Schnur, J. M.; Ratna, B. R.; Selinger, J. V.; Singh, A.; Jyothi, G.; Easwaran, K. R. K. Science 1994, 264, 945-947.

(43) Frankel, D. A.; O Brien, D. F. J. Am. Chem. Soc. 1994, 116, 10057-10069.

(44) Yui, H.; Minamikawa, H.; Danev, R.; Nagayama, K.; Kamiya, S.; Shimizu, T. Langmuir 2008, 24, 709-713.

(45) Terech, P.; Talmon, Y. Langmuir 2002, 18, 7240-7244.

(46) Chen, C. M. Phys. Rev. E 1999, 59, 6192-6195.

(47) Lubensky, T. C.; Prost, J. J. Phys. II 1992, 2, 371-382.

(48) Helfrich, W.; Prost, J. Phys. Rev. A 1988, 38, 3065-3068.

(49) Penfold, J.; Tucker, I.; Thomas, R. K.; Staples, E.; Schuermann, R. J. Phys. Chem. B 2005, 109, 10760-10770.

(50) Hayter, J. B. Langmuir 1992, 8, 2873-2876.

(51) Evans, D. F.; Mitchell, D. J.; Ninham, B. W. J. Phys. Chem. 1984, 88, 6344-6348. 